\title{
Health and Culture: Factors Influencing Immigrant Women's Health Beliefs and Health Behaviour
}

\author{
Alla Kushniryk, Mount Saint Vincent University, Canada \\ Stan Orlov, Mount Saint Vincent University, Canada \\ Emma Wertz, Kennesaw State University, U.S.A
}

\begin{abstract}
The purpose of this paper is to examine the impact of culture on immigrant women's health beliefs and attitudes toward health and cancer. The survey of 247 women was conducted in Canada and the USA. We found that acculturation played an important role in how immigrant women viewed cancer, and that their attitudes toward Western medicine and medical examinations changed with the amount of time they spent in North America. The role of family in health decision making was also found to be an important factor in health-related decisions for immigrant women.
\end{abstract}

Keywords: acculturation, culture, immigrant women's health, health beliefs, health behavior 


\section{Introduction}

It is impossible to underemphasize the influence of culture on the ways we communicate, evaluate and respond to health and illness. Individuals generally act in ways that correspond to cultural influences and expectations (Berry, Poortinga, Segall, \& Dasen, 1992). The concept of acculturation examines the extent to which individuals retain their culture when in a new country and various ways in which culture affects behaviour (Sam \& Berry, 2006). Cultural differences should be taken into consideration in order to better understand health behaviors of people from different cultural backgrounds (Trill \& Holland, 1994). The purpose of this paper is to investigate the impact of culture on immigrant women's health beliefs and attitudes toward health and cancer: Is acculturation a factor in shaping health behaviour? Do immigrant women change their health beliefs and attitudes to be more appropriate in their new society?

In this study, we use the modified Measure of Chinese Cultural Views of Health and Cancer (Liang et al., 2008). This instrument was revised to create a unified measure of cultural views on health and cancer which will help to understand the influence of different cultural views on health behaviour and decision making in cancer screening. Liang et al. (2008) stated:

Future cross-cultural research is needed to test the generalizability and specificity of these cultural constructs to other cultural groups. If this instrument can be used in other cultural groups, it is likely that programs to improve cancer screening adherence in these cultural groups could be combined in a culturally appropriate way (p. 372).

This study was conducted in two phases. During the first phase of research, we collected and analyzed twenty in-depth interviews with immigrant women. Liang's et al. (2008) instrument was revised based on the results derived from the setwenty qualitative interviews with immigrant women and the review of the literature on immigrant and minority women's health decision making. In the second phase, we collected and analyzed quantitative survey data.

In this paper, we hypothesize that cultural views on health and cancer consist of several measurable domains, and that acculturation plays an important role in shaping immigrant women's views on health and cancer. 


\section{Literature Review}

\section{Acculturation}

The concept of acculturation refers to the consequences of contact between distinct cultural groups (Berry, 1997; Sam \& Berry, 2006). It is often used to examine the degree to which immigrant populations maintain their culture in the receiving country and various ways in which the culture affects behavior (Sam \& Berry, 2006). According to Sam and Berry (2006), dimensions of culture, such as diversity, equality, conformity, wealth, space and time, have an impact on acculturation, with the likelihood of acculturation difficulties increasing with the difference in the aforementioned factors between the immigrant and the native populations. Health outcomes of ethnic populations have been of particular interest when examining acculturation and migration of individuals to new cultures (Lara, Gamboa, Kahramanian, Morales, \& Hayes Bautista, 2005).

Multiple studies looked at the relationship between acculturation and health. Studies of immigrant versus native health differences often find that recent immigrants, in general, fare better than non-immigrants and immigrants that have spent long time in the receiving country (Guendelman, 1998; Lee, O’Neill, Ihara \& Chae, 2013; McDonald \& Kennedy, 2004; Singh \& Siahpush, 2001; Stephen, Foote, Hendershot \& Shoenborn, 1994). This effect might be attributed, among other things, to healthier habits, including a lower intake of alcohol and nicotine (Blake, Ledsky, Goodenow \& O'Donnell, 2001; Chien, George \& Armstrong, 2002; Yoong et al., 2004). However, the more time immigrants spend in the receiving country, the more they converge with the native-born population (Ayala, Baquero \& Klinger, 2008; Blake, Ledsky, Goodenow \& O'Donnell, 2001; Maximova, O’Loughlin \& Gray-Donald, 2011; Singh $\&$ Siahpush, 2002).

Acculturation has also been associated with an elevated risk of obesity among immigrant populations. For example, Fuentes-Afflick and Hessol (2008) found that one measure of acculturation, length of time, was associated with obesity among childbearing Latina women in the United States. Acculturation has also been used as a concept to help better understand cancer information preferences among immigrant women (Thomson \& Hoffman-Goetz, 2009) and to examine body image, attitude and weight among Mexican Americans (Petti \&Cowell, 2011), and breast self-examination among Latina women (Peragallo, Fox, \& Alba, 2000). 
Acculturation has also been linked to poor dietary intake among South Asians (Lesser, Gasevic \& Lear, 2014), and Mexican-American women (Guendelman \& Abrams, 1995; Garcia-Maas, 1999). However, Weigers and Sherraden (2001) warned about oversimplifying the effect that acculturation might have on negative health behaviors of Mexican women when they pointed out the polytonic and multidimensional characteristics of acculturation.

\section{Fatalism}

Fatalism has been reported as one of the main barriers to breast and cervical cancer screening for many groups such as Latinas (Espinosa de los Monteros \& Gallo, 2011), Asian-Americans (Wu, Hsieh, \& West, 2008), Korean Americans (Lee, 2000), South-Asian women in the United States and Canada (Bottorff et al., 2001; Grewal, Bottorff, \& Hilton, 2005), Chinese (Liang at al., 2004; Liang et al., 2008), African Americans (Ashing-Giwa et al., 2004; Moy, Park, Feibelmann, Chiang, \& Weissman, 2006), Africans (Ghebre et al., 2014; Mayo, Hunter, \& Parker, 2003), Middle Easterners (Baron-Epel, Friedman, \& Lernau, 2009; Rashidi \& Rajaram, 2000), and women from the former Soviet Union (Remennick, 2003; Resick, 2008).

Ashing-Giwa et al. (2004) argued that some African-American, Asian and Latina women believe that cancer is contagious, is caused by breast trauma, and is a death sentence. Moy et al. (2006) noted that African Americans held a strong belief that cancer was fatalistic and ultimately deadly, and this belief was a reason for avoiding mammograms. It has also been documented that many women are reluctant to discuss cancer. For example, AfricanAmerican, Asian, and Latina women participants reported that older generations did not discuss breast health and Asian and Latina women mentioned that breast health was not an appropriate discussion topic (Moy et al., 2006).

Liang's Fatalism subscale consisted of nine items: 1. If I am meant to get cancer, I will get it; 2. No matter what I do, if I am going to get cancer, I will get it; 3. I cannot control my destiny; 4. Health or illness is a matter of fate. Some people are always healthy, others get sick very often; 5. Avoiding cancer is a matter of personal luck; 6. If I get cancer, the best way to deal with it is to accept it; 7. Getting cancer is like being sentenced to death; 8. It is best not to think about cancer. If we think about it too much, we probably will get cancer; 9. Bodily constitution is different for every person; therefore, some kinds of people are more likely to get cancer than others do. 
According to Liang et al. (2008), for Chinese women, the concept of fatalism is more closely aligned to "luck" and "destiny" than to religion and it should be tailored to fit a specific cultural group. We adopted all nine items from Liang's scale and added the following items: Whatever happens to us is in accordance with God's plan to emphasize the role of God in causing and curing cancer, and If I had cancer I would keep it secret from other people outside my family and close friends to measure reluctance to discuss cancer.

In this study, we hypothesize that:

H1: Acculturation plays an important role in how immigrant women view cancer. The immigrant women who lived less than 10 years in North America will score significantly higher on Fatalism subscale than those who lived more than 10 yearsand non-immigrant women.

\section{Attitude toward medical examinations}

Modesty can influence immigrant women's attitudes toward health and illness among various groups, such as Chinese women (Liang et al., 2008), Muslim women (Remennick, 2006), Mexican women (Wright, 2008), etc. Culture prevents open discussion about one's body and results in an un easiness with touching one's body, which has implications for regular breast screening (Ashing-Giwa et al., 2004). It was found that South-Asian women have fear and discomfort with showing breasts to a physician (Bottorff et al., 2001).Muslim women, specifically Arab women, have also been reported to experience a great concern over exposing their bodies (Hammoud, White \& Fetters, 2005; Remennick, 2006).

Liang's items 1 . I will be embarrassed if a doctor or a nurse checks my private parts; 2 . A lot of medical tests are too intrusive and make me uncomfortable; and 3. Medical doctors usually do unnecessary tests were used in this study to measure immigrant women's attitudes toward medical examinations. They were labelled Medical Examinations subscale. As a result, the researchers proposed that immigrant women would have more negative attitude toward medical examinations than non-immigrant women, which resulted in the following hypothesis:

$\mathrm{H} 2$ : Acculturation plays an important role on the immigrant women attitude toward medical examinations. The immigrant women who lived less than 10 years in North 
America will score significantly higher on Medical Examinations subscale than those who lived more than 10 years and non-immigrant women.

\section{Attitude toward Western medicine}

It is very important to understand the intersection of Western medicine and different cultural health approaches when considering the impact of culture on health behaviour. Several women interviewed in phase 1 of the study mentioned that herbal medicine was much healthier than Western medicine because herbs are made of natural ingredients. They were sometimes skeptical about Western medicine. Tome asure the attitude toward Western medicine, this study included the following items from Liang's Use of Herbs and Western Medicine subscales: 1. Herbs are a better remedy for illness than Western medicine; 2. Herbs are a better choice for preventing diseases than Western medicine;3. Western medicine is good for killing germs rather than preventing diseases; 4. We should not take "Western" medicine too often because its chemical ingredients will hurt our bodies.

As a result, it was hypothesized that immigrant women in this study would have more negative views about Western medicine than non-immigrant women and acculturation would be an important factor in changing these views:

H3: Acculturation plays an important role in how immigrant women view Western medicine. The immigrant women who lived less than 10 years in North America will score significantly higher on Western Medicine subscale than those who lived more than 10 years and non-immigrant women.

\section{Self-care}

Fear of cancer or denial of illness was also a common theme expressed among several cultural groups: South-East Asians (Gurm et al., 2008), Vietnamese Canadians (Donnelly, McKellin, Hislop, \& Long, 2009), Israeli-Muslim women (Remennick, 2006), Iranian women (Vahabi, 2010) and African Americans (Ashing-Giwa et al, 2004; Green, Lewis, Wang, Person, \& Rivers, 2004; Moy et al., 2006). Kleinman (1980) argued that when individuals deny illness, establishing a positive feedback relationship between disease and effective treatment becomes problematic. Women interviewed in phase 1 of the study, who immigrated from Africa, the Middle East, and Asia, mentioned that women (especially older women) in their home countries 
often do not seek medical attention unless they have a very serious illness like cancer or stroke. We included Liang's four Self-Care items in our survey: 1. As long as I can take good care of myself and keep myself healthy, I don't need to see a doctor; 2. I don't visit doctors if I'm not feeling sick; 3. I know my body better than anyone else; 4. Going to clinics or hospitals too often will cause me to catch diseases or having bad luck.

Taking into consideration this self-care factor, the following hypothesis was proposed:

H4: Acculturation plays an important role in how immigrant women view illness and self-care. The immigrant women who lived less than 10 years in North America will score significantly higher on Self-Care subscale than those who lived more than 10 years and non-immigrant women.

\section{Role of Family in Health Decision Making}

Family has been found to be a key influencer in women's individual health decisions across a wide range of cultures (Bottorff et al., 2001; Erwin et al., 2010; Grewal et al., 2005; Kleinman, 1980, Liang et al., 2004; Remennick, 2006). The immigrant women interviewed in phase 1 of this study highlighted the role played by family in supporting health decisions as a source of credibility, advice and/or affirmation. Family Approval items, which include: 1 . If I need to make any health related decisions I always discuss them with my family; and 2.It is very important to have family approval before undergoing any treatment were both derived from the previously collected qualitative data and added to the scale. They were included in the survey to measure the role of family in the immigrant women's health decision making.

Thus, it was hypothesized that:

H5: Family plays an important role in immigrant women's health decision making. The immigrant women who lived less than 10 years in North America will be more likely to seek Family Approval in health related issues thanthose who lived more than 10 years and non-immigrant women. 


\section{Methods}

\section{Interviews}

During the first phase of the study, we interviewed 20 immigrant women: six East-European women, four Asian women, six Middle-Eastern women, and four African women. The youngest participant was thirty-five and the oldest was sixty-two years old. All of the participants were married or had common-law partners and the majority had children. These interviews informed the creation of the survey which was used in the second phase of our research.

\section{Measures}

In the second phase of the study, we collected and analyzed quantitative survey data. The survey was administered in six languages: English, French, Spanish, Chinese, Russian, and Arabic. The survey consisted of the modified Measure of Chinese Cultural Views of Health and Cancer (Liang et al., 2008), demographic questions, questions about cultural views on health and illness, and attitudes toward cancer and cancer screening. Initially, women's cultural views were assessed by 24 items. Survey responses to each item were measured on a 5 -point Likert scale, ranging from strongly agree, agree, neutral, disagree, to strongly disagree.

Liang et al. (2008) proposed that Chinese cultural views involve at least seven cultural domains that may influence women's use of cancer screening tests to various degrees. These include Fatalism, Use of Herbs, Self-Care, Hot-Cold Balance, Lifestyle, Medical Examination, and Western Medicine. The subscale of Hot-Cold Balance is specifically relevant to Chinese culture and was not included in this study. However, the subscale Lifestyle was excluded from the analysis because it has general concepts of healthy lifestyle that a majority of participants would likely agree upon.

\section{Participants}

Criteria for selection of the participants were that women should have immigrated to Canada and the United States from Europe, Asia, Africa, and the Middle East and be aged between 18 and 70. The control group consisted of Canadian and American women who were born in North America and continued to live here. Purposive sampling was used to select participants according to the goals of the research. We identified the participants through the use of personal contacts in the community and with the help of immigrant settlement organizations. The 
researchers visited various locations and events where immigrant women gather, including churches, immigrant health fair, community centers, workshops and seminars for immigrants.

The survey was available in paper and online formats. Potential participants were invited to participate in the online survey via emails. We collected 140 valid paper surveys and 107 valid online surveys. Participants completed 175 surveys in English, 29 in Russian, 22 in Spanish, 12 in Arabic, 10 in French, and 4 in Mandarin. Even though the women were fluent in their native languages, many preferred the English version of the survey. Twenty-nine participants who completed the survey in Russian were born in the former USSR and are fluent in Russian, although their native languages included Ukrainian, Lithuanian, Belarusian, etc.Of the 247 participants, 41 (or 16.6\%) were 18-24 years of age, 22 (or $8.9 \%$ ) were 25-29 years of age, 25 (or $10.1 \%$ ) were $30-34$ years of age, 38 (or $15.4 \%$ ) were 35-39 years of age, 30(or 12.1\%) were 40-44 years of age, 22(or 8.9\%) were 50-54 years of age, 18 (or 7.5\%) were 55-59 years of age, and 24 (or $9.7 \%$ ) were 60 plus years of age.

In the survey, participants indicated their country of origin. The sample consisted of 175 immigrant women and 72 non-immigrant American and Canadian women. Immigrant women were classified by country of origin according to the geographical regions: 13 (5.3\%) women were born in Western Europe, 60 (24.3\%) in Eastern Europe, 35 (14.2\%) in the Middle East; 9 (3.6\%) in Africa, 12 (4.9\%) in South Asia, 14 (5.7\%) in Eastern Asia, (12.6\%) in Latin America, 37 (15\%) in USA, and 36 (14.6\%) in Canada. On average, the immigrant women in the study have lived in North America for 10 years, with the range from less than one year to 54 years. Ninety-four immigrant women lived less than 10 years in North America and 70 women more than 10 years. The majority of the women in the sample had a bachelor's (33.6\%) or graduate degree $(34.8 \%)$. One fourth $(25.9 \%)$ of women had a secondary (high) school graduation certificate or trades certificate or diploma.

\section{Results}

\section{Factor analysis}

During the first step in data analysis, the researchers utilized principal component factor analysis to identify the key factors that explain common and unique variances in the 24items that describe cultural views on health and illness. The scale is coded so that the higher scores represent higher traditional (as opposed to Western) cultural views on health and illness. 
Missing values were excluded from the analysis. This study used Verimax rotation option for factor rotations and to calculate interfactor correlations. Factors were extracted if their eigenvalues were greater than 1 . Items with loading values less than .4 were excluded from corresponding factors. Initially, eight factors were extracted as a result of the principal component analysis. The researchers examined the loading of items and evaluated the theoretical connection between items within factors. Items that loaded less than .4 in any of the factors or had theoretically weak associations with other items were eliminated from the scale:I know my body better than anyone else; Bodily constitution is different for every person, therefore, some kinds of people are more likely to get cancer than others do; If I get cancer, the best way to deal with it is to accept it; If I had cancer I would keep it secret from other people outside my family and close friends. As a result, 20 remaining items significantly loaded on six common factors: Fatalism, Western Medicine, Medical Examinations, Self-Care, Cancer Fear, and Family Approval (see Table 1). 
Table 1. Rotated Component Matrix ${ }^{a}$

\begin{tabular}{llllll} 
Factor & & & & & \\
1 & 2 & 3 & 4 & 5 & 6 \\
Fatalism Western & Medical & Self & Cancer & Family \\
& Medicine & Exams & Care & Fear & Approval \\
\hline
\end{tabular}

No matter what I do, if I am going to get cancer, I $\quad .834$

will get it.

If I am meant to get cancer, I will get it.

Health or illness is a matter of fate.

Some people are always healthy; others get sick $\quad .722$ very often.

I cannot control my destiny.

Avoiding cancer is a matter of personal luck.

Whatever happens to us is in accordance with

God's plan.

Herbs are a better choice for preventing diseases than Western medicine

Herbs are better remedy for illness than Western medicine.

We should not take "Western" medicine too often because its chemical ingredients will hurt our bodie

Western medicine is good for killing germs rather than preventing diseases.

\begin{tabular}{lc}
\hline A lot of medical tests are too intrusive and make & .791 \\
me uncomfortable. & .720 \\
Medical doctors usually do unnecessary tests. & .577 \\
I will be embarrassed if a doctor or a & \\
nurse checks my private parts.
\end{tabular}

As long as I can take good care of myself and keep

myself healthy, I don't need to see a doctor.

I don't visit doctors if I'm not feeling sick.

Going to clinics or hospitals too often will cause

me to catch diseases or having bad luck.

Getting cancer is like being sentenced to death.

It is best not to think about cancer. If we think

about it too much, we probably will get cancer.

If I need to make any health related decisions I

always discuss them with my family.

It is very important to have family approval before undergoing any treatment.

\footnotetext{
Extraction Method: Principal Component Analysis.

Rotation Method: Varimax with Kaiser Normalization.

a. Rotation converged in 6 iterations.
} 
The first factor contained six items and was labeled as Fatalism. Liang's et al. (2008) Fatalism subscale consisted of nine items. Two items from Liang's scale Getting cancer is like being sentenced to death and It is best not to think about cancer. If we think about it too much, we probably will get cancer were loaded on a new factor that were, then, labeled Cancer Fear.

Liang's Self-Care subscale included three items As long as I can take good care of myself and keep myself healthy, I don't need to see a doctor; I don't visit doctors if I'm not feeling sick; Going to clinics or hospitals too often will cause me to catch diseases or having bad luck that loaded on Self-Care factor. The item I know my body better than anybody else was eliminated from the subscale. Liang's instrument consisted of two subscales Use of Herbs and Western Medicine which consisted of two items each: Herbs are a better remedy for illness than Western medicine; Herbs are a better choice for preventing diseases than Western medicine from Liang's Use of Herbs subscale and Western medicine is good for killing germs rather than preventing diseases; We should not take "Western" medicine too often because its chemical ingredients will hurt our bodies. In our study, these four items loaded on one factor which was, then, labeled Western Medicine.

\section{Reliability}

The reliability (Cronbach's alpha) of the overall six-factor (20 items) scale was found to be .805 .

\section{Acculturation and Health Beliefs}

Hypothesis 1sought to determine whether acculturation plays a significant role in how immigrant women view cancer, and whether there was a significant difference in Fatalism between women who lived less than 10 years in North America, and those who lived more than 10 years, compared to non-immigrant women. A one-way Analysis of Variance (ANOVA) was calculated using Acculturation as an independent variable and Fatalism as a dependent variable. Variable Acculturation consisted of three groups: immigrant women who lived less than 10 years in North America, immigrant women who lived more than 10 years in North America, and non-immigrant women who were born in North America. A significant difference was noted: $F(2,216)=4.19, \mathrm{p}<.05$. In a follow-up to this test, a Tukey HSD post hoc was conducted. Table 2 examines where the exact differences were noted. 
Table 2. Post Hoc Analysis of Acculturation and Fatalism

\begin{tabular}{llllll}
\hline & N & M & SD & 10 plus years & Non-immigrants \\
1-9 years in North America & 87 & 19.68 & 5.99 & $*$ & $*$ \\
$\begin{array}{l}\text { 10 plus years in North } \\
\text { America }\end{array}$ & 65 & 17.31 & 5.76 & & NS \\
Non-immigrants & 64 & 17.46 & 5.25 & NS &
\end{tabular}

Note: $N S=$ non-significant difference between pair means; * = significance using the Tukey HSD procedure

Hypothesis 2 stated that immigrant women who lived less than ten years in North America will have more negative attitude toward medical examinations than immigrant women who lived more than 10 years and non-immigrant women. An ANOVA was calculated where Acculturation was an independent variable and Medical Examinations was a dependent variable. A significant difference was noted: $\mathrm{F}(2,227)=4.87, \mathrm{p}<.01$. In a follow-up to this test, a Tukey HSD post hoc was conducted. Table 3 examines where the exact differences were noted.

Table 3. Post Hoc Analysis of Acculturation and Medical Examinations

\begin{tabular}{llllll}
\hline & $\mathrm{N}$ & $\mathrm{M}$ & $\mathrm{SD}$ & 10 plus years & Non-immigrants \\
\hline 1-9 years & 88 & 8.13 & 2.49 & $*$ & $*$ \\
10 plus years & 70 & 7.07 & 2.25 & & NS \\
Non-immigrants & 69 & 7.20 & 2.23 & NS & \\
\hline
\end{tabular}

Note: NS = non-significant difference between pair means; * = significance using the Tukey HSD procedure

Hypothesis 3 posited that immigrant women would have more negative views about Western medicine than non-immigrant women and acculturation would be an important factor in changing these views. An ANOVA was calculated using Acculturation as an independent variable and Western Medicine as a dependent variable. A significant difference was not found: $\mathrm{F}(2,220)=1.98, \mathrm{p}>.05$. H3 was not supported.

Hypothesis 4 wanted to determine whether acculturation plays an important role in how immigrant women view illness and self-care and whether the immigrant women who lived less 
than 10 years in North America would score significantly higher on Self-Care subscale than those who lived more than 10 years and non-immigrant women. A significant difference was not found: $F(2,222)=1.70, \mathrm{p}>.05$. H4 was not supported.

Hypothesis 5 was designed to determine whether family played an important role in immigrant women's health decision making. It was hypothesized that the immigrant women who lived less than 10 years in North America would more likely seek family approval in health related issues than those who lived more than 10 years and non-immigrant women. An ANOVA was calculated where Acculturation was the independent variable and Family Approval was a dependent variable.

A significant difference was noted: $\mathrm{F}(2,228)=16.88, \mathrm{p}<.01$. In a follow-up to this test, a Tukey HSD post hoc was conducted. See Table 4 for the exact differences between groups.

Table 4. Post Hoc Analysis of Acculturation and Family Approval

\begin{tabular}{llllll}
\hline & $\mathrm{N}$ & $\mathrm{M}$ & $\mathrm{SD}$ & 10 plus years & Non-immigrants \\
\hline 1-9 years & 92 & 10.74 & 2.25 & $*$ & $*$ \\
10 plus years & 67 & 9.78 & 2.17 & & $*$ \\
Non-immigrants & 69 & 8.62 & 2.45 & $*$ & \\
\hline
\end{tabular}

Note: NS = non-significant difference between pair means; ${ }^{*}=$ significance using the Tukey HSD procedure

In this tudy, two items from Liang's Fatalism sub-scale were loaded on a new factor that was labeled Cancer Fear. An additional test (ANOVA) was run using Acculturation as the independent variable and Cancer Fear as a dependent variable. A significant difference was noted: $F(2,227)=13.39, \mathrm{p}<.01$. In a follow-up to this test, a Tukey HSD post hoc was conducted. Table 5 examines where the exact differences were noted. 
Table 5. Post Hoc Analysis of Acculturation and Cancer Fear

\begin{tabular}{llllll}
\hline & $\mathrm{N}$ & $\mathrm{M}$ & $\mathrm{SD}$ & 10 plus years & Non-immigrants \\
\hline 1-9 years & 90 & 5.0111 & 1.86327 & NS & $*$ \\
10 plus years & 68 & 4.4118 & 1.78915 & & $*$ \\
Non-immigrants & 69 & 3.5942 & 1.39662 & $*$ & \\
\hline
\end{tabular}

Note: $N S=$ non-significant difference between pair means; $*$ = significance using the Tukey HSD procedure

\section{Discussion}

This paper discusses findings of the study aiming to develop a measure of cultural views on health and illness. In this study, the researchersattempted to modify Liang's et al. (2008) Chinese cultural views on health and illness scale, in order to apply it to other cultural groups. The findings suggest that cultural views consist of at least six factors that describe cultural beliefs and attitudes toward health, illness and cancer. The original 23 -item Liang's scale consists of seven subscales: Fatalism, Use of Herbs, Self-Care, Hot-Cold Balance, Life Style, Medical Examination, and Western Medicine. In our attempt to modify the scale, six subscales emerged and captured important aspects of cultural views on health and illness: Fatalism, SelfCare, Medical Examinations, Cancer Fear, Western Medicine, and Family Approval. The subscales Hot-Cold Balance and Life Style were not included in the analysis. Hot-Cold Balanceis specifically relevant to Chinese culture. Life Style includes general concepts of healthy lifestyle that majority of participants would likely agree upon. The subscale Family Approval was added to the scale to capture the role of family in health decision making. The original Liang's 9-item Fatalism subscale was modified to a 6-item subscale and a 2-item subscale to measure levels of fatalism and fear of cancer in women from different cultural backgrounds. Fear of getting cancer was found to be a separate factor apart from fatalism.

This study sought to determine whether acculturation plays an important role in how immigrant women view cancer and Western medicine; if their attitudes toward medical examinations and self care change with time; and whether the role of family becomes less prominent in health related decisions as they live longer in North America. The study found a relationship between Fatalism and Acculturation. Immigrant women who lived less than ten years in North America scored significantly higher on Fatalism than those who lived more than 10 years and non- 
immigrant women who were born and lived in North America. This may mean that the belief that nothing can be done to prevent cancer significantly changes with the time spent in North America.

The study also revealed that level of cancer fear, i.e. the belief that cancer is deadly and should not be discussed, was considerably higher for the immigrant women in general. Both groups of immigrant women scored significantly higher than non-immigrants. However, the study did not support the relationship between acculturation and cancer fear. Similarly, the study did not display any relationship between acculturation and attitudes toward traditional and Western medicine, use of herbs, and self-care. In the future, it might be interesting to study how different cultural groups and age groups respond to these subscales.

One of the major findings of the study is the role of family in immigrant women's health decision making. It is important for immigrant women to discuss health related issues with the family and get family approval before making any major health decisions. This need to seek family approval diminishes with time spent in a new country, but the role of family in health related decisions still remains very important. This study found that immigrant women who lived less than ten years in North America will seek family approval more often than those who lived more than 10 years and more often than non-immigrant women; however, immigrant women who lived more than 10 years will more likely involve family in health decision making than non-immigrant women.

These findings are very important to those who seek to better understand the health choices made by women. Currently, a majority of breast and cervical cancer campaigns are targeted solely at women. As a result of this study, it is suggested that culturally relevant materials focus not only on immigrant women, but also on male family members. It is possible that husbands and fathers are influencing health choices of their female family members, and if they do not understand the importance and value of cancer screening, such influence might negatively affect women's health choices. It is recommended that more research should be done in this area. 
This study has several limitations. First, the generalizability of this study is limited by the use of the non-probability sampling strategy and the current size of the sample. Second, the cultural view scales were modified from "The Measure of Chinese Cultural Views of Health and Cancer" (Liang, et al., 2008) that were designed to measure Chinese cultural values. However, Liang et al. suggested that this instrument could be used for other cultural groups. In an attempt to do so, the researchers have conducted extensive research prior to administering the survey, in order to create a unified measure of cultural views on health and illness. It is possible that not all aspects are captured by the study's scale. Lastly, except for the Fatalism subscale, all subscales consist of 2 to 4 items, which may affect the results of the study. 


\section{References}

Ashing-Giwa, K. T., Padilla, G., Tejero, G., Kraemer, J., Wright, K., Coscarelli, A., Clayton, S., Williams, I., \& Hills, D. (2004). Understanding the breast cancer experience of women: A qualitative study of African American, Asian America, Latina and Caucasian cancer survivors. Psycho-Oncology, 13, 408-424. doi:10.1002/pon.750

Ayala, G. X., Baquero, B., \& Klinger, S. (2008). A systematic review of the relationship between acculturation and diet among Latinos in the United States: Implications for future research. Journal of the American Dietetic Association, 108(8), 1330-1344.

Baron-Epel, O., Friedman, N., \& Lernau, O. (2009).Fatalism and mammography in a multicultural population. Oncology Nursing Forum, 36(3), 353-361. doi:10.1188/09.ONF.353-361

Berry, J. W. (1997). Immigration, acculturation, and adaptation. Applied Psychology, 46(1), 5-34. doi:10.1111/j.1464-0597.1997.tb01087.x

Berry, J. W., Poortinga, Y. H., Segall, M. H., \& Dasen, P. R. (1992). Cross-cultural psychology: Research and applications. New York: Cambridge University Press.

Blake, S. M., Ledsky, R., Goodenow, C., \& O'Donnell, L. (2001).Recency of immigration, substance use, and sexual behavior among Massachusetts adolescents. American Journal of Public Health, 91(5), 794.

Bottorff, J. L., Johnson, J. L., \&Venables, L. J. (2001). Voices of immigrant South Asian women: Expressions of health concerns. Journal of Health Care for the Poor and Underserved, 12(4), 393-403. doi:10.1353/hpu.2010.0742

Chien, L. Y., George, M. A., \& Armstrong, R. W. (2002). Country of birth and language spoken at home in relation to illicit substance use.Canadian Journal of Public Health, 93(3), 188-92.

Donnelly, T. T., McKellin, W., Hislop, G., \&Long, B. (2009). Socioeconomic influences on Vietnamese-Canadian women's breast and cervical cancer prevention practices: A social determinant's perspective. Social Work in Public Health, 24, 454-476. doi:10.1080/19371910802678772

Erwin, D. O., Treviño, M., Saad-Harfouche, F. G., Rodriguez, E. M., Gage, E., \& Jandorf, L. (2010). Contextualizing diversity and culture within cancer control interventions for Latinas: Changing interventions, not cultures. Social Science \& Medicine, 71, 693701. doi:10.1016/j.socscimed.2010.05.005 
Espinosa de los Monteros, K., \& Gallo, L. C. (2011). The relevance of fatalism in the study of Latinas' cancer screening behavior: A systematic review of the literature.

International Journal of Behavioral Medicine, 18(4), 310-318. doi:10.1007/s12529010-9119-4

Fuentes-Afflick, E., \&Hessol, N. A. (2008). Acculturation and body mass among Latina women. Journal of Women's Health, 17(1), 67-73.

Garcia-Maas, L. D. (1999). Intergenerational analysis of dietary practices and health perceptions of Hispanic women and their adult daughters. Journal of Transcultural Nursing, 10(3), 213-219.

Ghebre, R. G., Sewali, B., Osman, S., Adawe, A., Nguyen, H. T., Okuyemi, K. S., \& Joseph, A. (2014). Cervical cancer: Barriers to screening in the Somali community in Minnesota. Journal of Immigrant and Minority Health, 1-7. doi:10.1007/s109030140080-1

Green, L., Lewis, R. K., Wang, M. Q., Person, S., \& Rivers, B. (2004). Powerlessness, destiny, and control: The influence on health behaviors of African Americans. Journal of Community Health, 29(1), 15-27. doi:0094-5145/04/0200-015/0

Grewal, S., Bottorff, J. L., \& Hilton, B. A. (2005).The influence of family on immigrant South Asian women's health. Journal of Family Nursing, 11, 242-263. doi:10.1177/1074840705278622

Guendelman, S. (1998). "Health and Disease Among Hispanics.” Pp. 277-301 in Handbook of Immigrant Health, Loue S. (Ed.) Springer.

Guendelman, S., \& Abrams, B. (1995). Dietary intake among Mexican-American women: Generational differences and a comparison with white non-Hispanic women. American Journal of Public Health, 85(1), 20-25.

Gurm, B. M., Joanne Stephen, J., MacKenzie, G., Doll, R. Barroetavenac, M. C., \& Cadell, S. (2008). Understanding Canadian Punjabi-speaking South Asian women's experience of breast cancer: A qualitative study.International Journal of Nursing Studies, 45, 266276. doi:10.1016/j.ijnurstu.2006.08.023

Hammoud, M. M., White, C. B., \& Fetters, M. D. (2005). Opening cultural doors: providing culturally sensitive healthcare to Arab American and American Muslim patients. American journal of obstetrics and gynecology, 193(4), 1307-1311. 
Kleinman, A. (1980). Patients and healers in the context of culture: An exploration of the borderland between anthropology, medicine, and psychiatry. Los Angeles, CA: University of California Press.

Lara, M., Gamboa, C., Kahramanian, M. I., Morales, L. S., \& Hayes Bautista, D. E. (2005). Acculturation and Latino health in the United States: A review of the literature and its sociopolitical context. Annual Review of Public Health, 26, 367-397.

Lee, M. C. (2000). Knowledge, barriers, and motivators related to cervical cancer screening among Korean-American women: A focus group approach. Cancer nursing, 23(3), 168-175.

Lee, S., O’Neill, A. H., Ihara, E. S., \& Chae, D. H. (2013). Change in self-reported health status among immigrants in the United States: Associations with measures of acculturation. Plos ONE, 8(10), 1. doi:10.1371/journal.pone.0076494

Lesser, I. A., Gasevic, D., \& Lear, S. A. (2014).The association between acculturation and dietary patterns of South Asian immigrants. Plos ONE, 9(2), 1-6. doi:10.1371/journal.pone.0088495

Liang, W., Wang, J. H., Chen, M., Feng, S., Lee, M., Schwartz, M. D., Pasick, R. J., \& Mandelblatt, J. S. (2008). Developing and validating a measure of Chinese cultural views of health and cancer. Health Education Behaviour, 35(3), 361-375. doi:10.1177/1090198106294893

Liang, W., Yuan, E., Mandelblatt., J. S., \&Pasick, R. J. (2004). How do older Chinese women view health and cancer screening? Results from focus groups and implications for interventions. Ethnicity \& Health, 9(3), 283-304. doi:10.1080/1355785042000250111

Maximova, K., O’Loughlin, J., \& Gray-Donald, K. (2011). Healthy weight advantage lost in one generation among immigrant elementary schoolchildren in multi-ethnic, disadvantaged, inner-city neighborhoods in Montreal, Canada. Annals of Epidemiology, 21(4), 238-244. doi:10.1016/j.annepidem.2011.01.002

Mayo, R. M., Hunter, A., \& Parker, V. G. (2003).Fatalism toward breast cancer among the women of Ghana. Health Care For Women International, 24(7), 608-616.

McDonald, J. T., \& Kennedy, S. (2004). Insights into the 'healthy immigrant effect': health status and health service use of immigrants to Canada. Social science \& medicine, 59(8), 1613-1627. 
Moy, B., Park, E. R., Feibelmann, S., Chiang, S., \& Weissman, J. S. (2006). Barriers to repeat mammography: Cultural perspectives of African American, Asian, and Hispanic women. Psycho-Oncology, 15, 623-634. doi:10.1002/pon.994

Peragallo, N. P., Fox, P. G., \& Alba, M. L. (2000).Acculturation and breast self-examination among immigrant Latina women in the USA. International Nursing Review, 47(1), $38-45$.

Petti, Y. M., \& Cowell, J. M. (2011).An assessment of measures of body image, body attitude, acculturation, and weight status among Mexican American women. Journal of the American Academy of Nurse Practitioners, 23(2), 84-91.

Rashidi, A., \& Rajaram, S. S. (2000) Middle Eastern Asian Islamic women and breast selfexamination: Needs assessment. Cancer Nursing, 23(1), 64-70.

Remennick, L. (2003). I have no time for potential troubles": Russian immigrant women and breast cancer screening in Israel. Journal of Immigrant Health, 5(4), 153-163.

Remennick, L. (2006). The challenge of early breast cancer detection among immigrant and minority women in multicultural societies.The Breast Journal, 12(1), 103-110. doi:10.1111/j.1075-122X.2006.00204.X

Resick, L. (2008). The meaning of health among midlife Russian-speaking women. Journal Of Nursing Scholarship, 40(3), 248-253. doi:10.1111/j.1547-5069.2008.00233.x

Sam, D. L., \& Berry, J. W. (Eds.). (2006). The Cambridge handbook of acculturation psychology. New York, NY: Cambridge University Press.

Singh, G. K., \& Siahpush, M. (2001).All-cause and cause-specific mortality of immigrants and native born in the United States. American Journal of Public Health, 91(3), 392.

Singh, G. K., \& Siahpush, M. (2002). Ethnic-immigrant differentials in health behaviors, morbidity, and cause-specific mortality in the United States: an analysis of two national data bases. Human Biology, 83-109.

Stephen, E., Foote, K., Hendershot, G., \& Shoenborn, C. (1994). Health of the foreign-born population: United States 1989-90. Advance data from vital and health statistics, 241, $1-12$.

Thomson, M. D., \& Hoffman-Goetz, L. (2009). Defining and measuring acculturation: a systematic review of public health studies with Hispanic populations in the United States. Social Science \&Medicine, 69(7), 983-991.

Trill, M. D., \& Holland, J. (1993). Cross-cultural differences in the care of patients with cancer: A review. General Hospital Psychiatry, 15(1), 21-30. 
Vahabi, M. (2010). Iranian women's perception and beliefs about breast cancer.Health Care for Women International, 31(9), 817- 830. doi:10.1080/07399331003725515

Weigers, M. E., \& Sherraden, M. S. (2001). A critical examination of acculturation: The impact of health behaviors, social support and economic resources on birth weight among women of Mexican descent. The International Migration Review, 35(3), 804839.

Wright, E. K. (2008). How Mexican-American women define health: Cultural beliefs and practices in a non-native environment (Unpublished doctoral dissertation), University of Tennessee, Knoxville.

Wu, T. Y., Hsieh, H. F., \& West, B. T. (2008). Demographics and perceptions of barriers toward breast cancer screening among Asian-American women. Women \& Health, 48(3), 261-281. doi:10.1080/03630240802463384

Yoong, W., Wagley, A., Fong, C., Chukwuma, C., \& Nauta, M. (2004). Obstetric performance of ethnic Kosovo Albanian asylum seekers in London: a case-control study. Journal of Obstetrics \& Gynecology, 24(5), 510-512. 